\title{
Photoperiodism in Hamsters: Abrupt Versus Gradual Changes in Day Length Differentially Entrain Morning and Evening Circadian Oscillators
}

\author{
Michael R. Gorman, ${ }^{* \neq, 1}$ David A. Freeman, $\$, 2$ and Irving Zucker ${ }^{*,+}$ \\ Departments of ${ }^{*}$ Psychology and ${ }^{\dagger}$ Integrative Biology, University of California, Berkeley, CA 94720; \\ ${ }^{\ddagger}$ Department of Psychology, University of Michigan, Ann Arbor, MI 48109; and \\ ${ }^{s}$ Department of Physiology and Neurobiology, University of Connecticut, Storrs, CT 06269
}

\begin{abstract}
In studies of photoperiodism, animals typically are transferred abruptly from a long (e.g., $16 \mathrm{~h}$ light per day [16L]) to a short (8L) photoperiod, and circadian oscillators that regulate pineal melatonin secretion are presumed to reentrain rapidly to the new photocycle. Among rats and Siberian hamsters, however, reentrainment rates vary depending on whether additional darkness is added to morning or evening, and a subset of hamsters (nonresponders) fails ever to reentrain normally to short photoperiods. The authors assessed whether several short-day responses occurred at different rates when darkness was extended into morning versus evening hours and the effectiveness of abrupt versus gradual shortening in day lengths (DLs). Entrainment patterns of photoresponsive hamsters also were compared to those of photononresponsive hamsters. Responsive hamsters transferred on a single day from $16 \mathrm{~L}$ to $8 \mathrm{~L}$ underwent more rapid gonadal regression, weight loss, decreases in follicle-stimulating hormone titers, and expansion of nocturnal locomotor activity when darkness was added to morning versus evening. When the dark phase was extended gradually by $8 \mathrm{~h}$ over 16 weeks, short-day responses occurred at the same rate whether darkness was appended to morning or evening or was added symmetrically. Darkness added to evening promoted more rapid short-day responses when it was added gradually rather than abruptly, despite the fact that average DLs were significantly shorter for the latter group. Among nonresponders, morning extensions of darkness transiently increased activity duration, whereas evening extensions did not. Gradual and abrupt decreases in DL differentially affect entrainment of evening and morning circadian oscillators. The authors argue for the incorporation of simulated natural photoperiods in studies of photoperiodism.
\end{abstract}

Key words testes, circadian rhythms, follicle-stimulating hormone, body weight, melatonin, seasonality

\section{INTRODUCTION}

The Siberian hamster, Phodopus sungorus, has been a useful model for studies of photoperiodism. When exposed to natural day lengths (DLs) of $50^{\circ} \mathrm{N}$ latitude, males undergo weight loss and gonadal regression and molt into a winter pelage as DLs decrease in late summer and autumn (Heldmaier and Steinlechner, 1981). Similar responses can be induced by transferring hamsters from long ( $16 \mathrm{~h}$ light $[16 \mathrm{~L}])$ to short $(8 \mathrm{~L})$

1. To whom all correspondence should be addressed. Present address: Department of Psychology and Michigan Society of Fellows, University of Michigan, Ann Arbor, MI 48109-1109; phone: (313) 764-5212; fax: (313) 763-7480; e-mail: mgorman @umich.edu.

2. Present address: Department of Psychology, University of California, Berkeley, CA 94720. 
photoperiods (Hoffmann, 1982). The physiological and behavioral changes induced by decreases in DL are mediated by increases in the duration of nocturnal secretion of melatonin (MEL) (Bartness and Goldman, 1988; Goldman, 1991), which is positively correlated with the length of the daily dark phase (Goldman et al., 1981; Illnerova, 1991). Both the absolute duration of nightly MEL secretion and changes in the duration of the nocturnal MEL signal influence photoperiodic responses (Bartness and Goldman, 1988; Goldman, 1991; Gorman and Zucker, in press-b; Karp et al., 1990).

It is commonly presumed, but rarely verified, that the duration of elevated nocturnal MEL secretion rapidly readjusts to a new steady state after a decrease in DL. In Siberian hamsters transferred from $16 \mathrm{~L}$ to $8 \mathrm{~L}$, however, the manner in which the night is lengthened affects the rate at which the MEL signal expands (decompresses) and the testes undergo regression (Hoffmann and Illnerova, 1986). Three weeks after the photoperiod was reduced from $16 \mathrm{~L}$ to $8 \mathrm{~L}$, the duration of nocturnal MEL secretion was longer when darkness was added to morning than when it was added to evening. Similarly, after 7 weeks in $8 \mathrm{~L}$, testis weights had decreased significantly from initial values when darkness was extended into the morning hours but not when extended into the evening (Hoffmann and Illnerova, 1986).

A subpopulation of hamsters transferred from long to short DLs, moreover, never undergoes expansion of the MEL signal, and such hamsters do not exhibit gonadal regression or other short-day traits (Puchalski and Lynch, 1986). The circadian systems of these animals differ from those of responders in having a longer free-running period $(\tau)$, different phase response curves to light, and decreased propensity to split into component oscillations in constant light (LL) (Puchalski and Lynch, 1988a, 1991a, 1991b). A two-oscillator model of circadian rhythms has been postulated to account for various circadian phenomena including, inter alia, differences in entrainment rates to evening versus morning extensions of darkness and the occurrence of nonresponsiveness (Hoffmann and Illnerova, 1986; Pittendrigh, 1974; Puchalski and Lynch, 1991a). Briefly, the evening oscillator $(E)$ is proposed to drive both onset of locomotor activity and onset of elevated nocturnal MEL secretion, whereas the morning oscillator (M) terminates both processes (Elliott and Tamarkin, 1994). Each oscillator has an intrinsic freerunning period $\left(E=\tau_{E}, M=\tau_{M}\right)$ with $\tau_{E}<\tau_{M}$; this difference in period allows lengthening of MEL secre- tion and $\alpha$ (duration of the active phase of the activity rhythm) in short photoperiods (Daan and Berde, 1978; Pittendrigh, 1974). The two oscillators can stably exist in a number of configurations or phase relations. The oscillators are not mutually independent but rather coupled, or interacting, which limits the phase relations they may obtain. In constant darkness (DD), the oscillators eventually assume a phase relation that produces a fixed long-duration activity and MEL signal; in splitting of locomotor activity rhy thms induced by LL, evening and morning components first free run before adopting a stable antiphase relationship. The particular stable phase relation adopted, moreover, determines the period of the composite free-running rhythm $\left(\tau_{\mathrm{EM}}\right)$, which is intermediate between the intrinsic periods of the two oscillators (Pittendrigh, 1974). Consequently, an increase or decrease in the period of either constituent oscillator will increase or decrease, respectively, the period of the composite free-running rhythm.

Nonresponsiveness is characterized by the maintenance of a typical long-day activity pattern to short DLs (Puchalski and Lynch, 1986, 1988a). Failure to properly entrain to short DLs may result from differences from responders in the intrinsic periods of $E$ or $M\left(\right.$ e.g., $\tau_{E}>\tau_{M}$ or $\tau_{E}>24 \mathrm{~h}$ ) or in coupling strength (e.g., $\tau_{\mathrm{E}}$ and $\tau_{\mathrm{M}}$ are unaltered but $\mathrm{E}$ cannot escape control by $M$ after exposure to long DLs). The latter possibility is suggested by findings that nonresponders can be converted to responders after daily MEL injections or exposure to $\mathrm{T}$ cycles (Puchalski and Lynch, 1988b, 1994). Cessation of either treatment does not result in a reversion to the nonresponder phenotype as would be predicted if nonresponsiveness were a consequence of variations in the intrinsic periods of $\mathrm{E}$ and $\mathrm{M}$. Increased coupling strength of circadian oscillators among nonresponders also is suggested by the observation that a higher light intensity is necessary to induce splitting in LL (Puchalski and Lynch, 1991b). The two-oscillator model described here, although useful for explaining photoperiodic phenomena, nonetheless remains hypothetical and alternative models have not yet been excluded.

All but a few studies of mammalian photoperiodism have been conducted under artificial laboratory conditions in which animals maintained under static DLs for several weeks or months are exposed to an abrupt shift in DL of 4-8 h. Animals in nature are exposed neither to invariant DLs (photoperiod varies sinusoidally, changing $1-5 \mathrm{~min} /$ day at $55^{\circ} \mathrm{N}$ latitude, except at the time of the solstices) nor to abrupt 


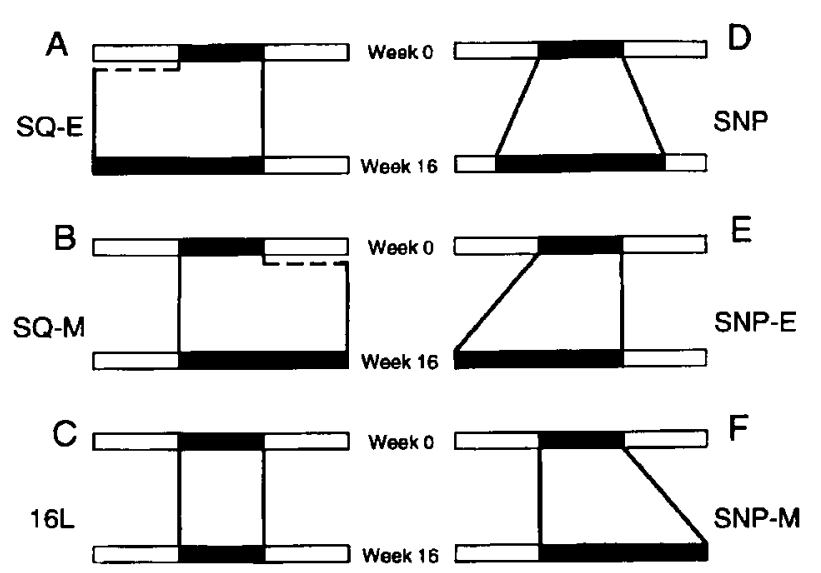

Figure 1. Schematic representation of photoperiods under which hamsters were maintained. Light and dark intervals are indicated by white and black bars, respectively. Until Week 0 , all hamsters were maintained in $16 \mathrm{~L}$. Animals were transferred to an $8 \mathrm{~L}$ photoperiod by (A) advancing abruptly the time of lights off (i.e., adding darkness to the subjective evening, SQ-E) or (B) delaying abruptly the time of lights on (i.e., adding darkness to morning, SQ-M). A control group (C) remained in 16L. In three other groups, darkness was extended gradually into $(D)$ both evening and morning as occurs in nature (SNP) or (E) exclusively into evening (SNP-E) or (F) morning (SNP-M). Abrupt transitions were effected in a single day, whereas gradual transitions were accomplished over 16 weeks.

changes in photoperiod. In presenting hamsters with more naturalistic photoperiodic stimuli, we have observed (a) a dissociation between DLs necessary for induction of gonadal regression and for the development of photorefractoriness (Gorman and Zucker, 1995b), (b) plasticity in the timing of photorefractoriness relative to gonadal regression (Gorman and Zucker, 1995a), and (c) accelerated photostimulation of growth under shorter but increasing DLs (Gorman, 1995). The present experiment assessed whether the different rates of induction of winter phenotypes that occur after abrupt morning versus evening extensions of darkness also would be obtained when DLs decreased gradually, as occurs in nature. We also directly compared rates of entrainment of circadian rhythms and photoinhibition among animals exposed to gradual versus abrupt changes in DL. Finally, we assessed whether failure of nonresponders to exhibit a decompressed entrainment pattern in short DLs resulted from differences in the periods of the constituent oscillators or from fixed coupling of the evening and morning oscillators.

\section{MATERIALS AND METHODS}

Male Siberian hamsters, $90-130$ days of age, were selected from a breeding colony maintained in a $16 \mathrm{~L}$ photoperiod (lights on 0200 PST). Hamsters were provided with food (Mouse Chow No. 5015, Purina Mills, St. Louis, MO) and tap water ad libitum and were housed in polypropylene cages $(27 \times 16 \times 13 \mathrm{~cm}$ high) on pine shavings (1-3 hamsters per cage). Cages were grouped in light-tight ventilated environmental chambers illuminated by two 30-watt fluorescent bulbs to produce a light intensity of 300-700 lux at the cage level.

Hamsters were randomly assigned at the beginning of the experiment (Week 0 ) to one of six groups $(n=$ 15-22 per group), five of which were transferred from $16 \mathrm{~L}$ to $8 \mathrm{~L}$ according to the following schedules. Two groups were exposed to an abrupt square-wave transfer from $16 \mathrm{~L}$ to $8 \mathrm{~L}$ accomplished on a single day at Week 0 either by adding the extra $8 \mathrm{~h}$ of darkness into the evening (SQ-E, lights on $0200 \mathrm{PST}, n=20$ ) (Fig. 1A) or morning (SQ-M, lights on $1000 \mathrm{PST}, n=21$ ) (Fig. 1B). In three groups, transfer from $16 \mathrm{~L}$ to $8 \mathrm{~L}$ was accomplished over 16 weeks by employing a simulated natural photoperiod (SNP) for $55^{\circ} \mathrm{N}$ latitude, accomplished nearly symmetrically as in nature (SNP, $n=15$ ) (Fig. 1D), or by appending extra darkness exclusively to the evening (SNP-E, $n=22$ ) (Fig. 1E) or morning (SNP-M, $n=19$ ) (Fig. 1F) hours. A control group remained in 16L $(n=16)$ (Fig. 1C).

\section{Measures}

Testis volume was estimated at Week 0 and at 4week intervals thereafter. Males were lightly anesthetized with methoxyflurane vapors (Metofane, Pitman Moore, St. Louis, MO), and length $(L)$ and width $(W)$ of the left testis were measured by an investigator unaware of the experimental treatment. Testis weight is approximated by a linear function of length times width squared, and this product was used as a measure of testis size (Gorman and Zucker, 1995a; WatsonWhitmyre and Stetson, 1985). Values were calculated and expressed as a percentage of the initial testis volume $\left(L W^{2} /\right.$ initial $\left.L W^{2} \times 100\right)$. While each hamster was anesthetized, approximately $0.5 \mathrm{ml}$ of blood was collected from the retroorbital sinus into nonheparinized tubes, centrifuged for $20 \mathrm{~min}$ at $5000 \mathrm{rpm}$, and plasma stored at $-40^{\circ} \mathrm{C}$ before being assayed for 
follicle-stimulating hormone (FSH) by radioimmunoassay (RIA). Serum plasma was measured with the NIAMDD rat FSH RIA kit using anti-rat FSH-10. The range of the assay (5\%-85\% total binding) was 1.0-80.7 $\mathrm{ng} / \mathrm{ml}$; samples that fell outside this range were assigned these limits as values. The lower limit was set at $5 \%$ rather than the conventional value $(15 \%)$, because the standard curve was not yet asymptotic at $15 \%$ and many of the values fell below the $15 \%$ limit. Body weights were measured at Week 0 and every 2 weeks thereafter, and similarly converted into percentages of initial body weights to facilitate analyses of changes over time.

A random subset of 7-9 hamsters in each group was housed singly in cages equipped with passive infrared motion detectors mounted externally above the cages. Movement across 3 or more of 27 zones activated a closed contact relay to Dataquest III software (MiniMitter, Sunriver, OR). Activity duration was determined weekly for each animal as follows. Activity counts for 10-min intervals were averaged over 7 days ( 5 days for Week 0 ) to generate a 24 -h histogram. Mean number of counts per 10 min over those 7 days (including both active and inactive intervals) was calculated for each animal. Activity onset and offset were defined as the first and last points, respectively, at which activity levels were $150 \%$ of the 10 -min average and sustained at that level for at least 3 of the next or last six 10 -min intervals. Activity duration $(\alpha)$ was obtained by subtracting time of onset from time of offset. $\alpha$ values that deviated more than $1.5 \mathrm{~h}$ in the same direction from both the immediately previous and subsequent values ( $2.1 \%$ of 867 observations) were replaced by the arithmetic mean of these latter values. Between Weeks 16 and 17, the previously unmonitored complement of hamsters was housed singly and monitored for 1 week, and these data were combined with those from Week 16 for analysis of final entrainment of the entire population. Activity records from each hamster were examined to determine whether activity onset coincided with lights off more closely than activity offset coincided with lights on; the former animals likely had $\tau s<24 \mathrm{~h}$, causing this group to be entrained by evening light, whereas $\tau$ s were likely $>24 \mathrm{~h}$ in the latter group, which was entrained by morning light.

Because not all Siberian hamsters are photoresponsive (Hoffmann, 1978; Puchalski and Lynch, 1986), some analyses were restricted to a subset of animals manifesting unambiguous gonadal and body weight responses to decreases in photoperiod. A decrease of $48 \%$ in testis volume or $12 \%$ in body weight from any previous value defined photoperiodic responses for these measures. These criteria were obtained from a separate study conducted under similar conditions (Gorman and Zucker, 1995a). At Week 24, paired testis weights were collected to verify that hamsters labeled nonresponders were not simply exhibiting delayed photoresponsiveness.

\section{Analyses}

Testis volumes, body weights, and activity durations were analyzed by four sets of repeated-measures analysis of variance (ANOVA, Statview 4.01, Abacus Concepts, Berkeley, CA) to highlight specific comparisons; Groups SQ-E and SQ-M were compared in an attempt to replicate the findings of Hoffmann and Illnerova (1986). The three SNP groups were compared to assess whether a comparable asymmetric effect of evening versus morning darkness would be obtained when extension of darkness occurred gradually. For both evening and morning extensions of darkness, we compared gradual and abrupt transitions to determine whether entrainment of activity rhythms and reproductive responsiveness would be enhanced in one condition. Finally, because FSH data violated assumptions of normality, these were analyzed with nonparametric tests; within-group comparisons were evaluated by Wilcoxon signed rank tests, pairwise comparisons between groups by Mann Whitney $U$ tests, and three-way between-group comparisons by the Kruskal-Wallis test. FSH values were analyzed only from hamsters demonstrating a photoperiodic testis response.

To assess whether nonresponders were characterized by stronger coupling of $\mathrm{E}$ and $\mathrm{M}$ oscillators or by altered free-running periods of these oscillators, $\alpha$ s of the continuously monitored sample were compared after evening extensions (SNP-E, SQ-E) and morning extensions (SNP-M, SQ-M) of darkness. We predicted that if increased coupling prevented expansion of $\alpha$,

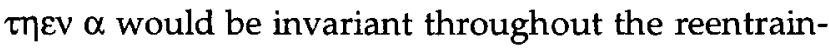
ment interval. If the compressed activity were a function of altered intrinsic periods of the $E$ and $M$ oscillators, then we expected to observe differences after evening and morning extensions of darkness (see Discussion section). 

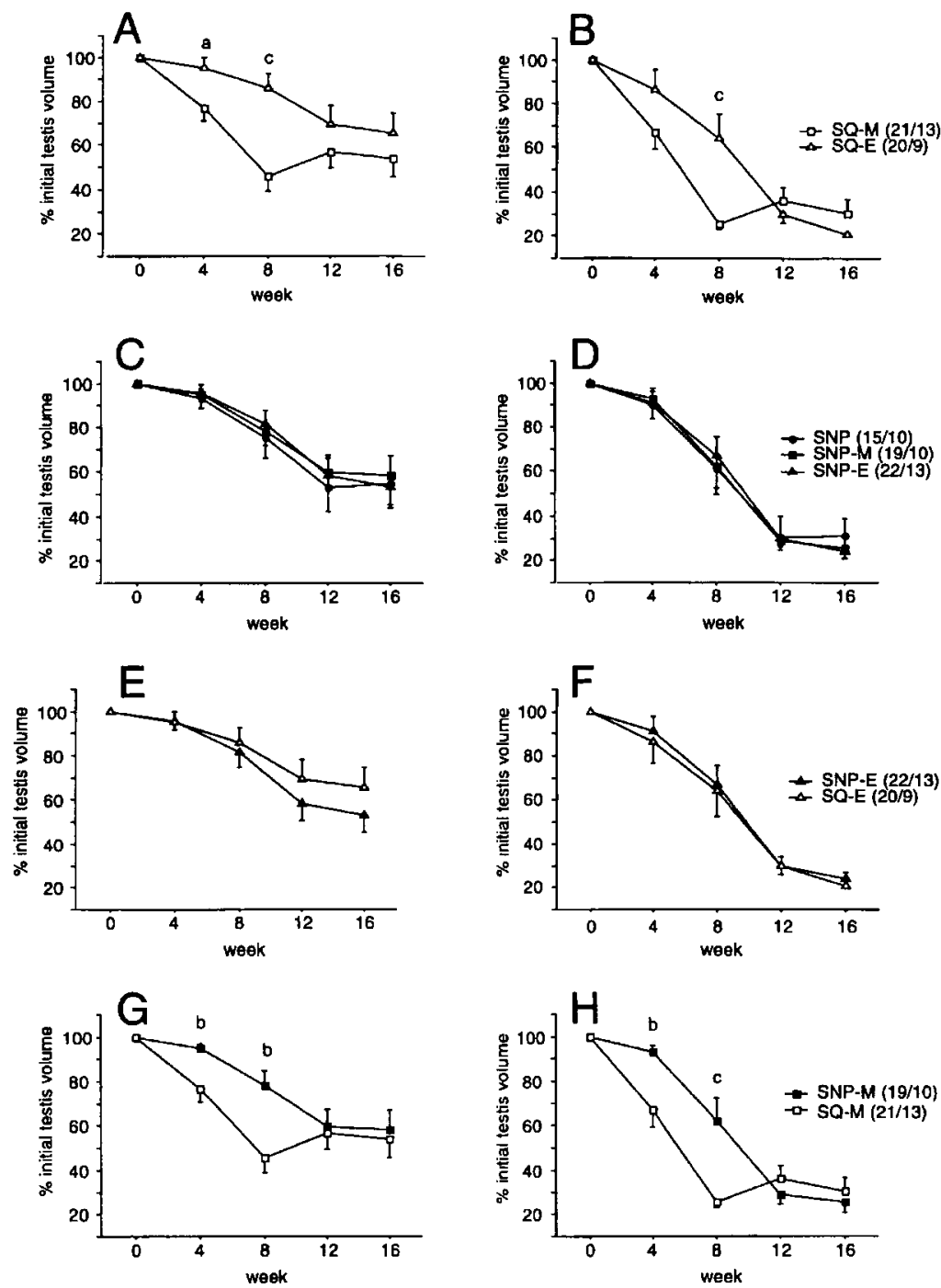

Figure 2. Mean $\pm S E M$ testis volume as a percentage of initial volume of all hamsters (left column: $A, C, E, G$ ) and photoresponsive hamsters (right column: B, D, F, H) exposed to experimental conditions. Data are multiply plotted to facilitate specific comparisons. Sample sizes are indicated in parentheses (all hamsters/photoresponsive hamsters). Letters indicate group means are significantly different (a: $p<.05$; b: $p<.01$; c: $p<.001$ ). Other abbreviations are as in Fig. 1 .

\section{RESULTS}

All four ANOVAs revealed main effects of the variable Time on testis volume and body weight $(p<.001$ in all cases by $F$ test), resulting from decreases in these measures over time in all groups except the one that remained in 16L. Differences between groups in the pattern of gonadal regression and weight loss are reflected in the interaction of the variables Time and Group. Only the results of this interaction are reported in what follows.

\section{SQ-M versus SQ-E}

Testis volume decreased significantly more rapidly in SQ-M than in SQ-E hamsters (interaction of Group and Time: $F=5.57, d f=4, p<.001$ ) (Fig. 2A). The former reached a minimum testis value at Week 8 , whereas SQ-E hamsters showed no significant decrease by this time but did thereafter. Testis volume was significantly lower in SQ-M than in SQ-E hamsters at Week $4(p<.05)$ and Week $8(p<.001)$ (Fig. 2A). A similar pattern was observed when analyses were restricted 
Table 1. Mean $\pm S E M$ follicle-stimulating hormone concentrations (nanograms/milliliter) of hamsters demonstrating a photoperiodic testis response to short day lengths and for all hamsters maintained in $16 \mathrm{~L}$.

\begin{tabular}{|c|c|c|c|c|c|}
\hline & Week 0 & Week 4 & Week 8 & Week 12 & Week 16 \\
\hline SQ-M $(n=12)$ & $\begin{array}{c}2.3 \pm 0.3 \\
(16)\end{array}$ & $\begin{array}{c}1.0 \pm 0.0^{\mathrm{a}, \mathrm{c}} \\
(100)\end{array}$ & $\begin{array}{c}2.0 \pm 0.6 \\
(75)\end{array}$ & $\begin{array}{c}1.2 \pm 0.1 \\
(84)\end{array}$ & $\begin{array}{c}1.4 \pm 0.3 \\
(75)\end{array}$ \\
\hline SQ-E $(n=9)$ & $\begin{array}{c}2.0 \pm 0.3 \\
\quad(11)\end{array}$ & $\begin{array}{c}1.4 \pm 0.2^{\mathrm{a}, \mathrm{b}} \\
(44)\end{array}$ & $\begin{array}{c}1.1 \pm 0.1 \\
(67)\end{array}$ & $\begin{array}{c}1.0 \pm 0.0 \\
(100)\end{array}$ & $\begin{array}{c}1.0 \pm 0.0 \\
(89)\end{array}$ \\
\hline $\operatorname{SNP}(n=9)$ & $\begin{array}{c}3.5 \pm 0.5 \\
(0)\end{array}$ & $\begin{array}{c}2.4 \pm 0.4 \\
\quad(22)\end{array}$ & $\begin{array}{c}1.1 \pm 0.2 \\
(67)\end{array}$ & $\begin{array}{c}1.3 \pm 0.3 \\
(78)\end{array}$ & $\begin{array}{c}1.2 \pm 0.2 \\
(78)\end{array}$ \\
\hline SNP-M $(n=9)$ & $\begin{array}{c}2.5 \pm 0.5 \\
\quad(11)\end{array}$ & $\begin{array}{c}2.1 \pm 0.4^{c} \\
(22)\end{array}$ & $\begin{array}{c}1.4 \pm 0.2 \\
(56)\end{array}$ & $\begin{array}{c}1.4 \pm 0.3 \\
(78)\end{array}$ & $\begin{array}{c}1.8 \pm 0.5 \\
(67)\end{array}$ \\
\hline SNP-E $(n=12)$ & $\begin{array}{c}2.9 \pm 0.4 \\
(8)\end{array}$ & $\begin{array}{c}2.6 \pm 0.7^{b} \\
(8)\end{array}$ & $\begin{array}{c}2.2 \pm 1.0 \\
(59)\end{array}$ & $\begin{array}{c}2.3 \pm 0.9 \\
(67)\end{array}$ & $\begin{array}{c}2.3 \pm 1.0 \\
(75)\end{array}$ \\
\hline $16 \mathrm{~L}(n=20)$ & $\begin{array}{c}3.0 \pm 0.3 \\
(5)\end{array}$ & $\begin{array}{c}2.7 \pm 0.4 \\
(10)\end{array}$ & $\begin{array}{c}2.5 \pm 0.3 \\
(30)\end{array}$ & $\begin{array}{c}2.8 \pm 0.4 \\
\quad(15)\end{array}$ & $\begin{array}{l}2.8 \pm 0.4 \\
\quad(15)\end{array}$ \\
\hline
\end{tabular}

NOTE: Percentage of samples below lower limit of the assay is indicated under each mean value in parentheses. Means marked with the same letter differ significantly-a: $p<.005 ; \mathrm{b}: p<.05 ; \mathrm{c}: p<.001$. Abbreviations are as in Fig. 1.

to the subset of photoresponsive hamsters; testis volume decreased more rapidly in SQ-M than in SQ-E hamsters $(F=8.23 ; d f=4 ; p<.001)$ (Fig. 2B) and was smaller at Week 8 in SQ-M males than in SQ-E males $(p<.001)$ (Fig. 2B).

FSH profiles of hamsters manifesting a photoperiodic testis response paralleled changes in testis size; FSH concentrations at Week 4 decreased from initial values in SQ-M $(p<.01)$ but not in SQ-E hamsters $(p>$ .35) and were significantly lower in SQ-M than in SQ-E hamsters at this time $(p<.005)$ (Table 1$)$. All hamsters in SQ-M had undetectable FSH concentrations at Week 4 , whereas the corresponding value for SQ-E animals was $44 \%\left(\chi^{2}=8.8, p<.005\right)$. FSH was undetectable in all hamsters in SQ-E at Week 12, however, prior to the nadir in testis size at Week 16.

Body weights of SQ-E hamsters changed little over 16 weeks, whereas those of SQ-M hamsters decreased from Weeks 2 to 6 before increasing again (interaction of Group and Time: $F=2.49, d f=16, p<.005$ ) (Fig. 3A). Percentage initial body weight was significantly lower in SQ-M than in SQ-E hamsters at Week 1 and from Weeks 3 to $8(p<.05)$ (Fig. 3A). Among photoresponsive animals, body weights decreased first in SQ-M and later in SQ-E hamsters, resulting in a significant interaction of Group and Time ( $F=2.80, d f=16, p<$ .001) (Fig. 3B). Body weights were lower at Week 1 and Weeks 3-8 in SQ-M than in SQ-E photoresponsive hamsters $(p<.05)$ (Fig. 3B).

\section{SNPs}

Neither average testis volume over 16 weeks nor the pattern of decrease in testis size differed among hamsters exposed to SNP, SNP-E, or SNP-M (main effect: $p>.80$; interaction: $p>.90$ ) (Fig. 2C). The same result was obtained when only photoresponsive hamsters were considered ( $p>.90$ for both tests) (Fig. 2D).

FSH concentrations did not differ between SNP groups at any time. SNP groups exhibited a decrease in FSH concentrations from initial values at Week $8(p<$ .05 for SNP and SNP-M, $p=.06$ for SNP-E) (Table 1), with undetectable FSH concentrations in the majority of animals from Week 8 onward (Table 1).

The pattern of decrease in body weight did not differ among SNP groups when all hamsters were considered $(p>.90)$ (Fig. 3C) or when analyses were restricted to photoresponsive hamsters $(p>$ .05) (Fig. 3D).

\section{SNP-E versus SQ-E}

Testis volume was similar among hamsters exposed to SNP-E and SQ-E $(p>.40)$ (Fig. 2E) and decreased at the same rate over time $(p>.40)$. The same result was obtained among photoresponsive hamsters from these two groups ( $p>.60$ for both tests) (Fig. 2F).

FSH values decreased from initial values at approximately the same time; hormone concentrations 

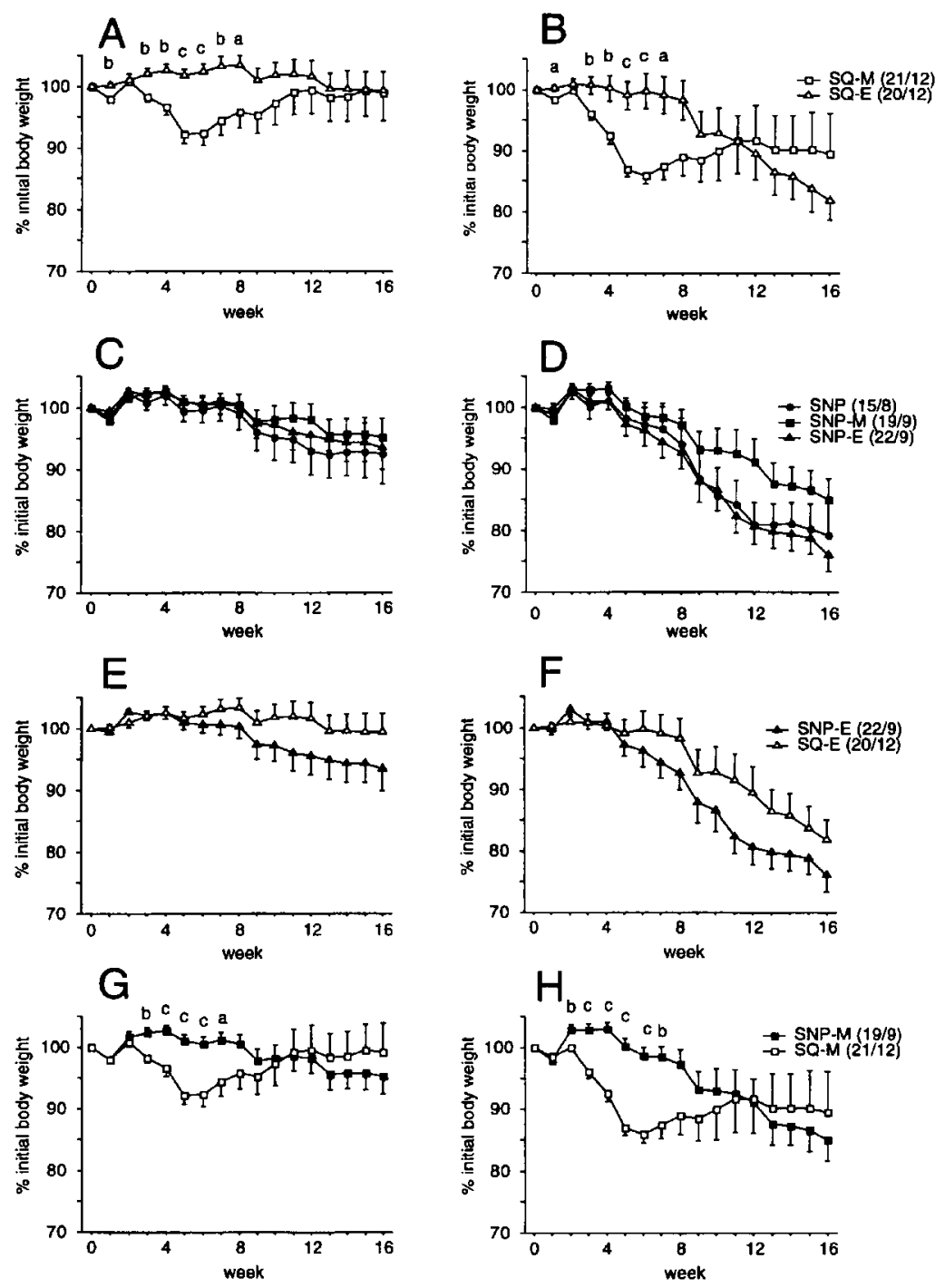

Figure 3. Mean $\pm S E M$ body weight as a percentage of initial body weight of all hamsters (left column: $A, C, E, G$ ) and photoresponsive hamsters (right column: B, D, F, H) exposed to experimental conditions. Sample sizes are indicated in parentheses (all hamsters/photoresponsive hamsters). Conventions and abbreviations are as in Figs. 1 and 2.

in SQ-E hamsters were not significantly lower than initial values until Week $8(p<.01)$, whereas a similar trend was evident in SNP-E hamsters $(p=.06)$. Nor were significant differences observed at any point in the percentage of hamsters with undetectable $\mathrm{FSH}$ concentrations $(p>.05)$. FSH titers, however, were significantly lower in SQ-E than in SNP-E hamsters at Week $4(p<.05)$.

Mean body weights of the entire sample did not differ among groups $(p>.20)$, but the Time $\times$ Group interaction was significant $(F=1.92, d f=16, p<.05)$ (Fig. 3E). The same result was obtained when only hamsters with a photoperiodic body weight response were considered $(F=2.44, d f=16, p<.005)$ (Fig. 3F).
Weight loss occurred more rapidly in SNP-E than in SQ-E responders; slopes of least squares regression lines were more negative in the former group $(p<.05)$.

\section{SNP-M versus SQ-M}

Testis volume decreased more rapidly in hamsters exposed to SQ-M than SNP-M $(F=4.82, d f=4, p<.005)$ (Fig. 2G) with lower values in the former group at Weeks $4(p<.005)$ and $8(p<.005)$. Among photoresponsive hamsters, a main effect of group failed to reach significance $(p<.06)$, but SQ-M hamsters underwent more rapid testicular regression than did SNP-M hamsters $(F=9.01, d f=4, p<.001)$ (Fig. $2 \mathrm{H})$. Testis 


\section{A}

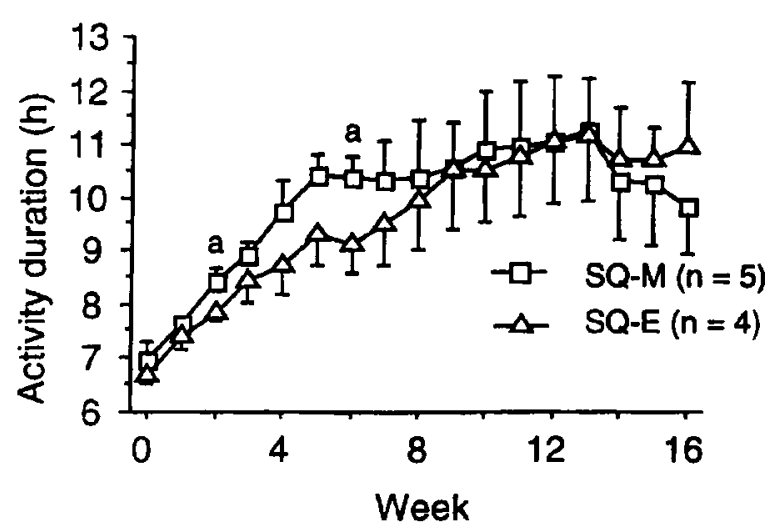

C

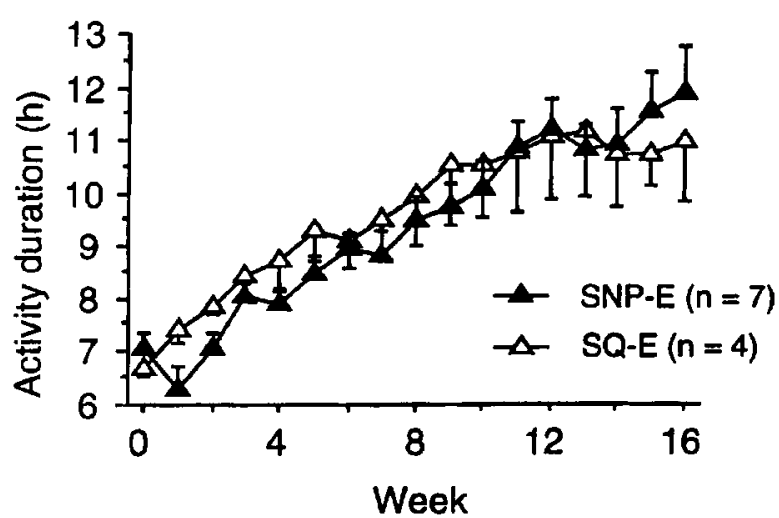

B

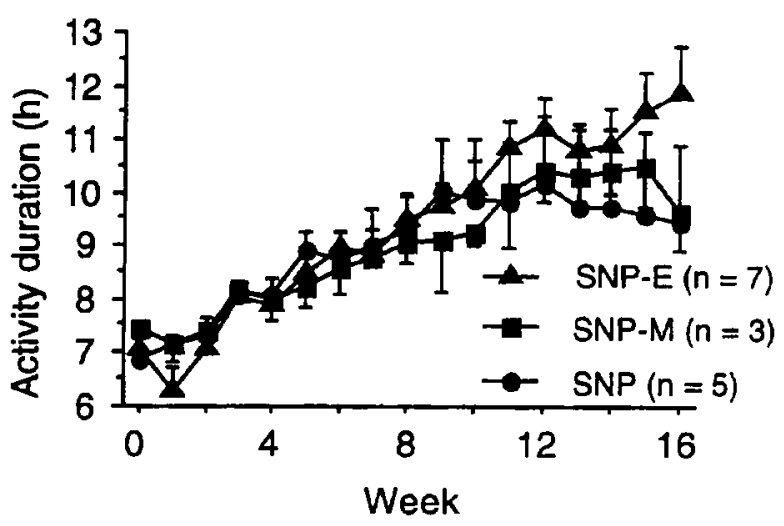

D

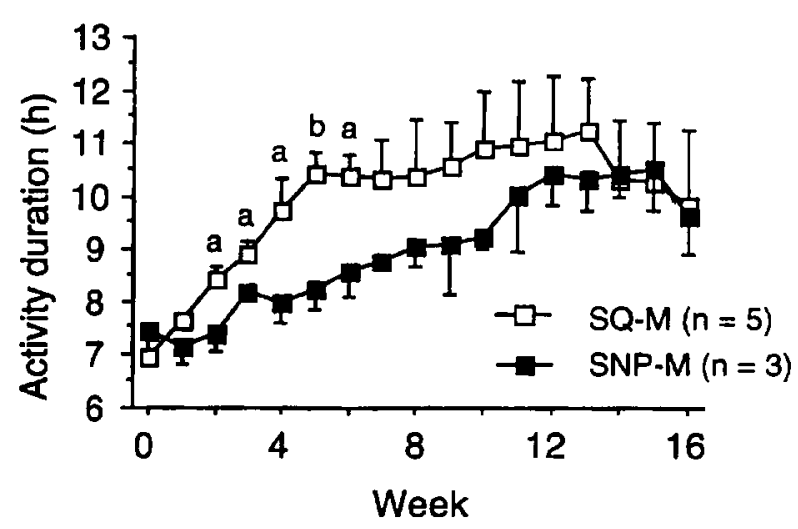

Figure 4. Mean $\pm S E M$ activity duration $(\alpha)$ of hamsters demonstrating a photoperiodic testis response from each experimental group plotted to facilitate specific comparisons. Sample sizes are indicated in parentheses. Abbreviations are as in Figs. 1 and 2.

volume of responders was likewise lower in the former group at Weeks $4(p<.01)$ and $8(p<.001)$.

Differences in FSH concentrations mirrored those observed in testis volume; FSH values decreased from initial values by Week 4 in SQ-M hamsters $(p<.01)$, but not until Week 8 in SNP-M hamsters $(p<.05)$, and were significantly lower in the former than the latter group at Week $4(p<.005)$ (Table 1). Moreover, a higher percentage of SQ-M than SNP-M hamsters had undetectable FSH concentrations at Week $4\left(\chi^{2}=14.0, p<\right.$ .001 ), but groups were similar at subsequent time points.

The pattern of decrease in body weight differed between groups $(F=3.38, d f=16, p<.001$ ) (Fig. 3G) with SQ-M hamsters undergoing an early decrease followed by a rise. SQ-M hamsters weighed signifi- cantly less than SNP-M hamsters from Weeks 3 to 7 $(p<.05)$ (Fig. 3G). SNP-M hamsters, by contrast, exhibited only later decreases in body weight. The same pattern was observed in photoresponsive hamsters, and the interaction of Group and Time was significant $(F=3.02, d f=16, p<.001)$ (Fig. $3 \mathrm{H})$. Groups differed between Weeks 2 and $7(p<.05)$ (Fig. 3H).

\section{Locomotor Activity}

Among photoresponsive hamsters monitored continuously, $\alpha$ increased with time in all groups $(p<.05$ by $F$ test). On account of the small sample size due to the large number of nonresponders, the four planned between-group ANOVAs revealed main effects of 


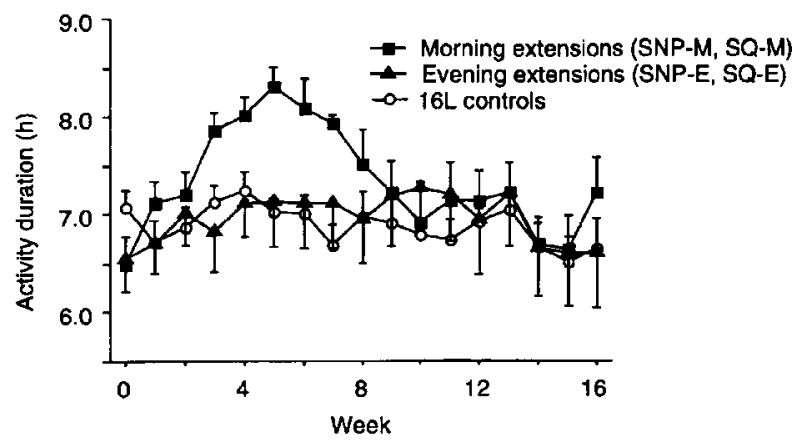

Figure 5. Mean $\pm S E M \alpha$ of morning-entrained nonresponsive hamsters after exposure to morning (SNP-M, SQ-M; $n=8$ ) or evening (SNP-E, SQ-E; $\boldsymbol{n}=8$ ) extensions of darkness. $\alpha$ 's of control hamsters maintained throughout in 16L $(n=9)$ are included for comparison.

Time but no significant main effect of Group or interactions of Group and Time (Fig. 4A-D). Guided by group differences in testis size, we conducted select pairwise comparisons of $\alpha$ around time points in which gonad size diverged. Among responders, $\alpha$ was greater in SQ-M than in SQ-E hamsters at Weeks 2 and $6(p=.05$, one-tailed) (Fig. 4A). $\alpha$ was significantly greater in SQ-M than in SNP-M hamsters at Weeks 2-6 $(p<.05$, one-tailed) (Fig. 4D).

$\alpha s$ of nonresponsive hamsters (all groups combined) varied significantly over time $(F=5.0, d f=16$, $p<.001)$, increasing between Weeks 0 and $6(p<.005)$ and decreasing again at Week 16 compared to Week 6 $(p<.005)$. All nonresponders were either morning entrained $(34 / 40)$ or could not be clearly classified as morning or evening entrained $(6 / 40)$, thus precluding separate analysis of evening entrainers. The majority of responsive hamsters also was entrained to morning $(41 / 55)$, although a minority was clearly entrained to evening $(3 / 55)$, and others $(11 / 55)$ were not readily classifiable as evening or morning entrained. Because the sample of continuously monitored hamsters was limited in size, gradual and abrupt morning groups were combined for analysis, as were gradual and abrupt evening groups. Among continuously monitored morning-entrained nonresponders, $\alpha$ increased nearly $2 \mathrm{~h}$ between Weeks 0 and 5 ( $p<.001$ ) (Fig. 5) when darkness was extended into morning (SNP-M, SQ-M). $\alpha$ subsequently decreased between Weeks 5 and $16(p<.05)$ (Fig. 5). By contrast, after evening extensions, morning-entrained nonresponders showed relatively fixed $\alpha$ s that did not differ from those of control hamsters maintained in $16 \mathrm{~L}$ throughout (Fig. 5). The model proposed later suggests that SQ-M nonresponders would undergo more rapid $\alpha$ expansion than would SNP-M nonresponders. Although standard errors were large, this prediction was supported as $\alpha$ was $0.5 \mathrm{~h}$ longer in SQ-M than in SNP-M hamsters at Weeks 3-6 (data not shown).

Among the entire sample monitored at Week 16, final $\alpha$ did not differ between groups exposed to the various $8 \mathrm{~L}$ conditions $(p>.95)$ (data not shown), nor did $\alpha$ differ when only responders were considered $(p>$ .85). Among the continuously monitored cohort, $\alpha s$ of photoresponsive males (all groups combined) were longer $(F=49.8, d f=1, p<.001)$ (Fig. 6A) and increased more rapidly over time $(F=23.51, d f=16, p<.001)$ (Fig. 6A) than did those of nonresponsive hamsters.

\section{Photoresponsiveness}

Paired testis weights at Week 24 were bimodally distributed, as would be expected in a population composed of responsive and nonresponsive hamsters (Fig. 6B). Only 1 hamster labeled a nonresponder on the basis of testis volume during the first 16 weeks had a paired testis weight $<550 \mathrm{mg}$ at Week 24 (Fig. 6B), indicating that these animals were not simply delayed in their gonadal response to photoperiod. The high incidence of nonresponsiveness, moreover, is comparable to values in previous experiments from our laboratory (Gorman and Zucker, 1995a, 1995b) and may result from housing animals in long DLs from birth (Gorman and Zucker, in press-a). Reproductive inhibition was confirmed in most hamsters labeled responsive, although a number exhibited signs of spontaneous recrudescence by this time (Fig. 6B). Finally, the incidence of photoperiodic testis and body weight responses did not vary among groups exposed to short photoperiods (testis response: $\chi^{2}=1.7, d f=4$, $p>.7$; body weight response: $\chi^{2}=3.8, d f=4, p>.4$ ).

\section{DISCUSSION}

Testicular involution, decreases in FSH concentrations, and body weight loss occurred significantly earlier when Siberian hamsters were transferred abruptly from long to short DLs by extending darkness into morning (SQ-M) than into evening (SQ-E) hours; these changes were evident within 4 weeks of transfer and maximal within 8 weeks in males in SQ-M, but were initiated only around Week 8 among hamsters in SQ-E. 
A

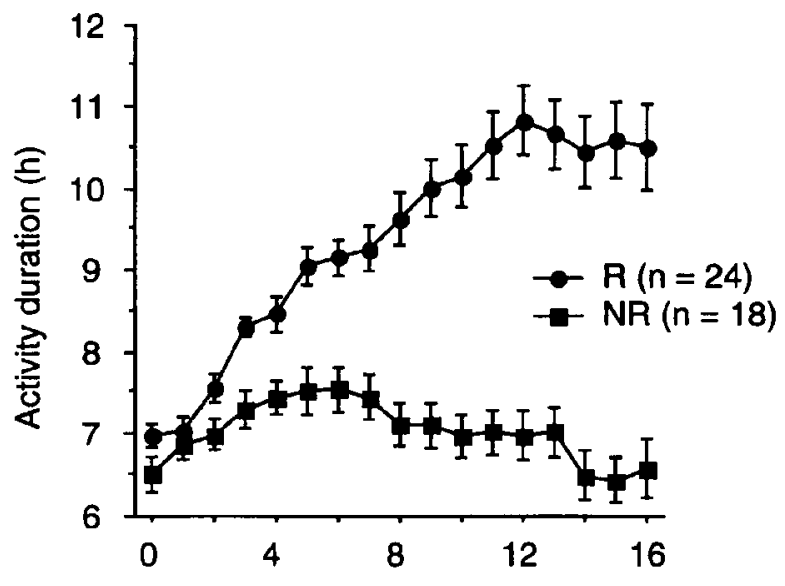

B

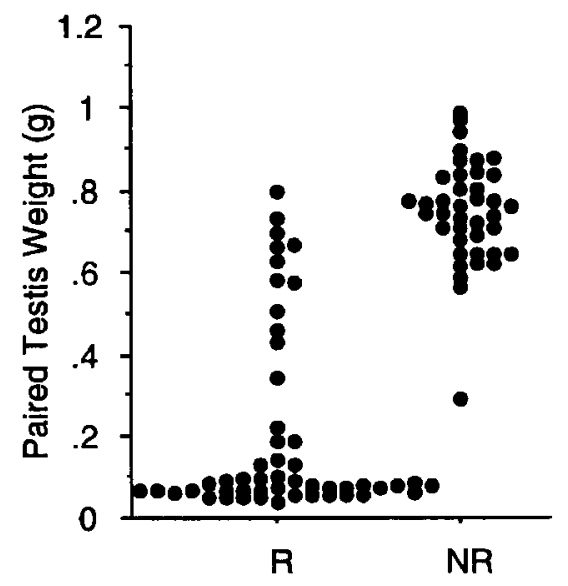

Figure 6. (A) $\alpha$ of hamsters demonstrating a photoperiodic testis response (R) or nonresponse (NR). Experimental groups were combined. Sample sizes are in parentheses. (B) Paired testis weights at 24 weeks of hamsters labeled responders $(R ; n=51)$ or nonresponders $(N R ; n=40)$ on the basis of testis volume measures at Weeks 0-16. Data from 5 hamsters are missing due to 2 deaths and the inadvertent failure to record testis weights from 3 animals.

Similar relations were obtained, whether the entire group was considered or just those animals that eventually exhibited photoperiodic responses. Activity duration $(\alpha)$ in SQ-M and SQ-E responsive hamsters, moreover, diverged between Weeks 2 and 6 when differences in testis volume, body weight, and FSH titers were first apparent, although small sample size precluded a robust test of these trends. These findings replicate and extend those of Hoffmann and Illnerova (1986), who demonstrated differences in rates of testis regression and the duration of nocturnal MEL secretion under similar conditions. This pattern differs from that observed in male Syrian hamsters in which morning and evening extensions of darkness produce similar results (Hastings et al., 1987).

By contrast, transitions between long and short DLs accomplished by gradual extensions of darkness into morning or evening produced similar rates of gonadal regression, body weight loss, decreases in FSH titers, and expansion of $\alpha$. Under conditions of gradually changing DLs, changes in dawn and dusk apparently are processed equally efficiently by the morning and evening oscillators.

Direct comparison between abrupt and gradual extensions of darkness into evening led to unexpected findings; gradual transfer to short DLs decreased body weight more effectively than did an abrupt transfer, even though the photophases always were longer in the former case and therefore expected to be less inhibitory. In Syrian hamsters, photoperiod-induced changes in body weight are pineal independent in some but not other experiments (Bartness and Wade, 1984), although this independence has not been demonstrated in Siberian hamsters. In the present case, the more rapid loss in body weight may reflect more rapid entrainment in SNP-E versus SQ-E hamsters. When activity data of responders and nonresponders were analyzed together, the interaction of Time and Group was highly significant $(F=2.9, d f=16, p<.001)$, with more rapid expansion in SNP-E than in SQ-E hamsters, although $\alpha$ did not differ significantly between groups at any time point. Further work with larger sample sizes would be required to confirm that the greater body weight reduction in SNP-E than in SQ-E hamsters is attributable to more efficient entrainment in the former group.

SNP-E and SQ-E hamsters did not differ on other variables measured less frequently than body weight. Both FSH concentrations and testicular volume decreased from initial values around Week 8 . Hamsters in the gradual transition treatment, however, were exposed to DLs above the putative critical photoperiod of 13L-14L (Duncan et al., 1985; Hoffmann, 1982) for approximately 4-6 weeks longer than were those transferred abruptly to $8 \mathrm{~L}$. If entrainment to their respective photoperiods occurred rapidly, males in SNP-E might be expected to undergo gonadal regression much later than those in SQ-E rather than concurrently, as was found here. Although FSH values were significantly lower in SQ-E than in SNP-E hamsters at Week 4 , this resulted in large part from inclusion of an 
outlier among the SNP-E group. The overall similarity between groups, along with the body weight data, suggests that the circadian system integrates gradual transitions in DL more efficiently or more completely than abrupt extensions of darkness into evening.

By contrast, when darkness was extended to morning hours, abrupt transitions led to more rapid gonadal and body weight responses than did gradual ones. Gonadal regression and weight loss in SNP-M hamsters were as complete as those in SQ-M hamsters, but lagged in the former group by about 4-6 weeks. These results would be predicted if entrainment were equally efficient under these conditions. Because hamsters in SNP-M are exposed to DLs above the putative critical photoperiod for several weeks, gonadal regression and weight loss should occur later than in hamsters in which circadian rhythms entrained rapidly to $8 \mathrm{~L}$ after an abrupt transition. Consistent with this interpretation, $\alpha$ was longer in SQ-M than in SNP-M hamsters between Weeks 2 and 6, although differences disappeared with time.

In summary, abrupt and gradual asymmetric transfers from long to short days did not produce similar results; whereas abrupt transfers achieved by extending darkness into the morning resulted in more rapid reproductive and body weight responses than did extensions into the evening hours, no differences in testis volume or FSH concentrations were noted after gradual extensions into morning versus evening. Gradual extensions of darkness into evening, however, led to more rapid weight loss than was observed with gradual extensions of darkness into morning.

The underlying circadian bases of differences in entrainment rates still remain to be elucidated. In the absence of entraining light cues (i.e., DD), the $E$ and $M$ oscillators move apart under the influence of their respective free-running periods, $\tau_{\mathrm{E}}$ and $\tau_{\mathrm{M}}$. The nature of the coupling varies as a function of the phase relation and produces a limit on the maximum expansion of elevated MEL secretion and locomotor activity (Elliott and Tamarkin, 1994). For $\alpha$ to expand as it does in responders, $\tau_{\mathrm{E}}$ must be $<\tau_{\mathrm{M}}$. Models suggest that a composite free-running period at a given phase relation, $\tau_{\mathrm{EM}}$ is intermediate between $\tau_{\mathrm{E}}$ and $\tau_{\mathrm{M}}$ (Pittendrigh, 1974). In the present experiment, among both responders and nonresponders, the preponderance of morning-entrained hamsters suggests that $\tau_{\mathrm{EM}}>24 \mathrm{~h}$ and, by implication, that $\tau_{\mathrm{M}}>24 \mathrm{~h}$ as well. Others have reported overall $\tau s>24 \mathrm{~h}$ and large angles of entrainment (morning entrainers) in nonresponsive hamsters, and $\tau s<24 \mathrm{~h}$ and small angles of entrainment (evening entrainers) in responsive Siberian hamsters (Puchalski and Lynch, 1986, 1991a). This discrepancy between responders in the present results and other studies may derive from differences between animal colonies or in the form of activity measured; we used motion detectors to measure a global form of activity, whereas others monitored wheel running.

Entrainment to long DLs forces the $\mathrm{E}$ close to the $\mathrm{M}$ oscillator by delaying the former and advancing the latter (Illnerova, 1991). Addition of darkness to evening (i.e., removal of evening light), therefore, would be expected to diminish or eliminate the delaying effects of light on $\mathrm{E}$ of responders and allow $\mathrm{E}$ to advance if $\tau_{\mathrm{E}}<24 \mathrm{~h}$ (Fig. 7A) (the case where $\tau_{\mathrm{E}}>24 \mathrm{~h}$ is discussed subsequently). By contrast, addition of morning darkness (i.e., removal of morning light) should diminish or eliminate the advancing effects on $\mathrm{M}$ of responders and allow it to be delayed (given that $\tau_{\mathrm{M}}>24 \mathrm{~h}$ ) relative to its previous position (Fig. 7B). That SQ-M led to more rapid short-day responses than did SNP-M suggests that the progressive delaying of $M$ was constrained by the rate of change in lights on (morning) in SNP-M and, therefore, that $\tau_{M}$ may be greater than the rate at which lights on was delaying in this condition (Fig. 7B). By contrast, the similarity of response in SQ-E and SNP-E suggests that $\tau_{\mathrm{E}}$ was not shorter than the rate at which lights off (evening) was advancing in SNP-E (Fig. 7A). Because the delays in lights on in SNP-M were equivalent to the advances in lights off in SNP-E (Fig. 1), the greater divergence of $\tau_{M}$ than $\tau_{E}$ from $24 \mathrm{~h}\left(\tau_{\mathrm{M}}-24>24-\tau_{\mathrm{E}}\right)$ would predict more rapid expansion of $\alpha$ and consequently more rapid short-day responses after abrupt morning versus evening transfers (Fig. 7A,B). With symmetrical coupling between oscillators, the greater divergence of $\tau_{\mathrm{M}}$ than $\tau_{\mathrm{E}}$ from $24 \mathrm{~h}$ also may result in an overall free-running period $\left(\tau_{\mathrm{EM}}\right)>24 \mathrm{~h}$, accounting for the finding that virtually all responsive and nonresponsive hamsters were morning entrained. In several species, delaying phase shifts induced by light or other treatments are achieved more rapidly than are advancing phase shifts (Illnerova, 1991). With abrupt changes in DL, entrainment may be achieved more rapidly when darkness is extended into morning rather than evening simply because the latter requires delays and the former advances of circadian oscillators.

Analysis of entrainment patterns in terms of intrinsic periods of $E$ and $M$, moreover, may offer the simplest explanation for the transient increase in $\alpha$ seen in nonresponsive hamsters after morning but not eve- 
A

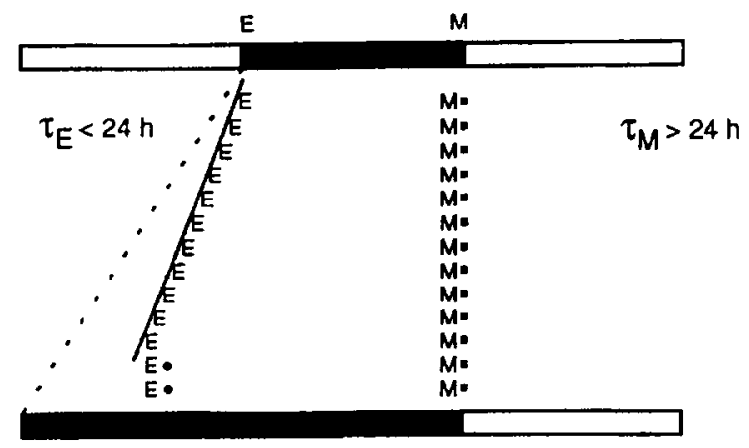

B

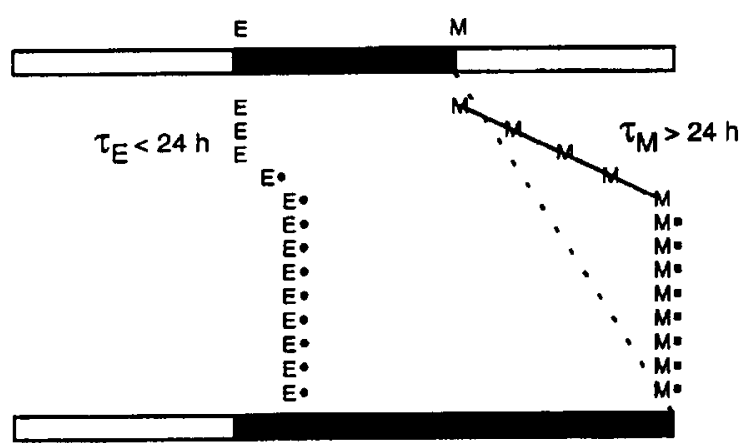

C

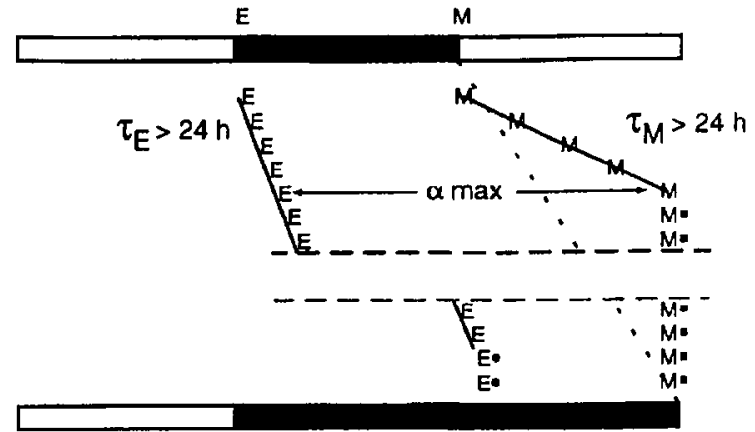

D

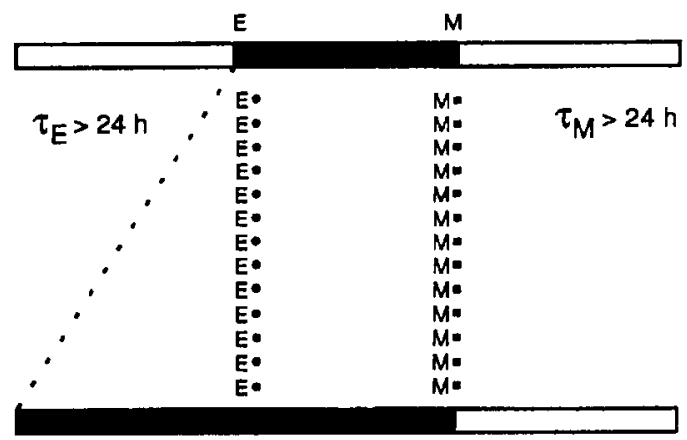

Figure 7. Schematic representation of movements of evening (E) and morning (M) oscillators. Black and white bars reflect times of darkness and light, respectively. In each panel, the upper and lower bars represent the initial and final photoperiod conditions, respectively. Dashed oblique lines indicate the trajectory of lights on or off under gradually changing conditions. Small black squares indicate that the position of the $\mathrm{M}$ oscillator is constrained by light onset. Small black circles indicate that the position of the $\mathrm{E}$ oscillator is constrained principally by strong coupling with the $M$ oscillator that occurs when $\alpha$ is near its maximum. (A) Among responders exposed to extensions of evening darkness $\tau_{E}<24 \mathrm{~h}$ and $\tau_{M}>24 \mathrm{~h}$ cause $M$ to remain apposed to lights on and $E$ to free run with a period (solid black line) longer than the rate by which lights off is advanced in SNP-E. Gradual and abrupt transfers will be expected to produce similar results. (B) Among responders, after morning extension of darkness, $M$ runs with a period longer than the rate by which lights on is delayed in SNP-M until it abuts lights on, at which point it remains fixed. E remains abutted against lights off until increased coupling with $M$ resulting from changing phase relations pulls (delays) E. Only the abrupt transfer case is depicted here. Gradual changes would cause $M$ to follow light onset. (C) Among nonresponders exposed to morning extensions of darkness, both $\mathrm{E}$ and $\mathrm{M}$ free run with $t>24 \mathrm{~h}$ until $\mathrm{M}$ abuts lights on. $E$ continues to free run until increased coupling with $M$ associated with a compressed phase relation prevents $E$ from delaying further. $\alpha$ reaches its maximum after $M$ has fully delayed but before $E$ has caught up to $M$. Dashed horizontal lines represent passage of time in which $E$ continues to free run before catching up with $M$. Only the abrupt transfer case is depicted. $M$ would follow light onset under gradual transfer conditions. (D) Among nonresponders, after evening extensions of darkness, the position of $M$ is fixed against lights on whereas the position of $E$ remains fixed due to coupling with $M$. Gradual and abrupt transfers would produce similar results. See text for additional details.

ning extensions of darkness. After morning extensions of darkness, the $M$ oscillator free runs with $\tau_{M}>24 \mathrm{~h}$. If $\tau_{\mathrm{E}}<\tau_{\mathrm{M}}$, then $\alpha$ will increase either because $\mathrm{E}$ is delaying less than is $M\left(\tau_{\mathrm{E}}>24 \mathrm{~h}\right.$ ) (Fig. $7 \mathrm{C}$ ) or because E cannot advance beyond the time of lights on $\left(\tau_{\mathrm{E}}<24\right.$ h) (Fig. 7B). Once the M oscillator again abuts the new lights on time, however, its position will be fixed by the time of lights on. If $\tau_{\mathrm{E}}>24 \mathrm{~h}$, then $\alpha$ will recompress
(Fig. 7C). If $\tau_{\mathrm{E}}<24 \mathrm{~h}$, then no recompression will occur and animals will be photoresponsive. The apparent dominance of $\mathrm{M}$ in determining phase angle of entrainment derives from the likelihood that $\tau_{\mathrm{EM}}>24 \mathrm{~h}$. After evening extensions (Fig. 7D), the $\mathrm{M}$ oscillator will be constrained by light onset as all nonresponders are morning entrained. Moreover, if $\tau_{\mathrm{E}}<24 \mathrm{~h}$ (Fig. 7A), then animals will successfully entrain and hence be 
responders. If $\tau_{\mathrm{E}}>24 \mathrm{~h}$, then the $\mathrm{E}$ oscillator also will be fixed by its coupling to the $M$ oscillator, which is itself fixed by lights on (Fig. 7D). This model suggests that the fundamental difference between responders and nonresponders may be that $\tau_{\mathrm{E}}<24 \mathrm{~h}$ in the former and $\tau>24 \mathrm{~h}$ in the latter. Because the intrinsic period of an oscillator cannot be measured in the absence of coupling from other oscillators that affect its expressed period, it is difficult to determine whether this model is distinct from that proposed by Puchalski and Lynch (1991a), who stressed the importance of asymmetric coupling favoring the $\mathrm{M}$ oscillator in nonresponders. Although transient increases in $\alpha$ were not previously reported for nonresponders transferred to short DLs (symmetrical transfers), Puchalski and Lynch's model might account for this phenomenon as a temporary loss of dominant coupling by $\mathrm{M}$ that requires time to reassert control over $\mathrm{E}$. The model proposed here, however, further predicts that nonresponders transferred to DD should undergo $\alpha$ expansion and exhibit short-day responses. Nonresponders were not tested under DD in the present experiment, but animals nonresponsive to short DLs underwent gonadal regression in DD in one colony (Freeman and Goldman, 1997) but not another (Puchalski and Lynch, 1988a, 1991a). Nonresponsiveness in different colonies or perhaps among individuals in a single cohort may have different circadian bases. Indeed, responders of other investigators were evening entrained (Puchalski and Lynch, 1991a) in contrast to the present results, attesting to differences between populations.

Our findings indicate that reentrainment of circadian rhythms differs markedly when DL decreases abruptly in fixed photoperiod regimens versus when DL decreases more gradually as in nature. We may infer that the rhythms of pineal MEL secretion also are differentially affected by the manner in which photoperiodic transfers are accomplished (Elliott and Tamarkin, 1994; Hoffmann and Illnerova, 1986). Many photoperiodic relations elaborated with artificial abrupt DL challenges may benefit from a reevaluation that incorporates simulated natural changes in DL. This seems to be an essential step if we are to understand the basis of photoperiodic regulation of seasonal rhythms under field conditions.

\section{ACKNOWLEDGMENTS}

This work was supported by National Institutes of Health (NIH) Grant HD-02982, a Berkeley fellowship, and the Michigan Society of Fellows. We are grateful to Bruce Goldman for assistance with the FSH assay supported by NIH Grant MH49818, Kimberly Pelz and Christiana Tuthill for excellent technical assistance, and Brian Prendergast, Theresa Lee, and Benjamin Rusak for comments on the manuscript.

\section{REFERENCES}

Bartness TJ and Goldman BD (1988) Peak duration of serum melatonin and short-day responses in adult Siberian hamsters. Am J Physiol 255:R812-R822.

Bartness TJ and Wade GN (1984) Photoperiodic control of body weight and energy metabolism in Syrian hamsters (Mesocricetus auratus): Role of pineal gland, melatonin, gonads and diet. Endocrinology 114:492-498.

Daan S and Berde C (1978) Two coupled oscillators: Simulations of the circadian pacemaker in mammalian activity rhythms. J Theor Biol 70:297-313.

Duncan MJ, Goldman BD, DiPinto MN, and Stetson $\mathrm{MH}$ (1985) Testicular function and pelage color have different critical daylengths in the Djungarian hamster, Phodopus sungorus sungorus. Endocrinology 116:424-430.

Elliott JA and Tamarkin L (1994) Complex circadian regulation of pineal melatonin and wheel-running in Syrian hamsters. J Comp Physiol A 174:469-484.

Freeman DA and Goldman BD (1997) Evidence that the circadian system mediates photoperiodic nonresponsiveness in Siberian hamsters: The effect of running wheel access on photoperiodic responsiveness. J Biol Rhythms 12:100-109.

Goldman BD (1991) Parameters of the circadian rhythm of pineal melatonin secretion affecting reproductive responses in Siberian hamsters. Steroids 56:218-225.

Goldman BD, Hall V, Hollister C, Reppert S, Roychoudhury P, Yellon S, and Tamarkin L (1981) Diurnal changes in pineal melatonin content in four rodent species: Relationship to photoperiodism. Biol Reprod 24:778-783.

Gorman MR (1995) Seasonal adaptations of Siberian hamsters. I. Accelerated gonadal and somatic development in increasing versus static long day lengths. Biol Reprod 53:110-115.

Gorman MR and Zucker I (1995a) Seasonal adaptations of Siberian hamsters. II. Pattern of change in day length controls annual testicular and body weight rhythms. Biol Reprod 53:116-125.

Gorman MR and Zucker I (1995b) Testicular regression and recrudescence without subsequent photorefractoriness in Siberian hamsters. Am J Physiol 269:R800-R806.

Gorman MR and Zucker I (in press-a) Environmental induction of photononresponsiveness in the Siberian hamster, Phodopus sungorus. Am J Physiol.

Gorman MR and Zucker I (in press-b) Pattern of change in melatonin duration determines testicular responses in Siberian hamsters, Phodopus sungorus. Biol Reprod.

Hastings MH, Walker AP, and Herbert J (1987) Effect of asymmetrical reductions of photoperiod on pineal melatonin, locomotor activity and gonadal condition of male Syrian hamsters. J Endocrinol 114:221-229. 
Heldmaier G and Steinlechner S (1981) Seasonal control of energy requirements for thermoregulation in the Djungarian hamster (Phodopus sungorus), living in natural photoperiod. J Comp Physiol B 142:429-437.

Hoffmann K (1978) Effects of short photoperiods on puberty, growth and moult in the Djungarian hamster (Phodopus sungorus). J Reprod Fertil 54:29-35.

Hoffmann K (1982) The critical photoperiod in the Djungarian hamster Phodopus sungorus. In Vertebrate Circadian Systems: Structure and Physiology, J Aschoff, S Daan, and G Gross, eds, pp 297-304, Springer, New York.

Hoffmann K and Illnerova H (1986) Photoperiodic effects in the Djungarian hamster: Rate of testicular regression and extension of pineal melatonin pattern depend on the way of change from long to short photoperiods. Neuroendocrinology 43:317-321.

Illnerova $H$ (1991) The suprachiasmatic nucleus and rhythmic pineal melatonin production. In Suprachiasmatic $\mathrm{Nu}$ cleus: The Mind's Clock, DC Klein, RY Moore, and SM Reppert, eds, pp 197-216, Oxford University Press, New York.

Karp JD, Dixon ME, and Powers JB (1990) Photoperiod history, melatonin, and reproductive responses of male Syrian hamsters. J Pineal Res 8:137-152.

Pittendrigh CS (1974) Circadian oscillations in cells and the circadian organization of multicellular systems. In The Neurosciences: Third Study Program, FO Schmitt and FG Worden, eds, pp 437-458, MIT Press, Cambridge, MA.
Puchalski W and Lynch GR (1986) Evidence for differences in the circadian organization of hamsters exposed to short day photoperiod. J Comp Physiol A 159:7-11.

Puchalski W and Lynch GR (1988a) Characterization of circadian function in Djungarian hamsters insensitive to short day photoperiod. J Comp Physiol A 162:309-316.

Puchalski W and Lynch GR (1988b) Daily melatonin injections affect the expression of circadian rhythmicity in Djungarian hamsters kept under a long-day photoperiod. Neuroendocrinology 48:280-286.

Puchalski W and Lynch GR (1991a) Circadian characteristics of Djungarian hamsters: Effects of photoperiodic pretreatment and artificial selection. Am J Physiol 261:R670R676.

Puchalski W and Lynch GR (1991b) Expression of circadian rhythmicity in Djungarian hamsters under constant light: Effects of light intensity and the circadian system's state. J Comp Physiol A 169:185-189.

Puchalski W and Lynch GR (1994) Photoperiodic time measurement in Djungarian hamsters evaluated from T-cycle studies. Am J Physiol 267:R191-R201.

Watson-Whitmyre M and Stetson MH (1985) Mathematical method for estimating paired testes weight from in situ testicular measurements in three species of hamsters. Anat Rec 213:473-476. 